\title{
Revaluation of Mbelek and Lachièze-Rey scalar tensor theory of gravitation to explain the measured forces in asymmetric resonant cavities
}

\author{
F. O. Minotti ${ }^{1,2}$
}

\begin{abstract}
The scalar-tensor theory of gravitation proposed by Mbelek and Lachiè ze-Rey has been shown to lead to a possible explanation of the forces measured in asymmetric resonant microwave cavities [1]. However, in the derivation of the equations from the action principle some inconsistencies were observed, like the need no to vary the electromagnetic invariant in a scalar source term. Also, the forces obtained were too high, in view of reconsideration of the experiments originally reported and of newly published results [2]. In the present work the equations are re-derived using the full variation of the action, and also the constant of the theory re-evaluated employing the condition that no anomalous gravitational effects are produced by the earth's magnetic field. It is shown that the equations originally employed were correct, and that the newly evaluated constant gives the correct magnitude for the forces recently reported

\footnotetext{
${ }^{1}$ Universidad de Buenos Aires, Facultad de Ciencias Exactas y Naturales, Departamento de Física, Buenos Aires, Argentina.

${ }^{2}$ CONICET-Universidad de Buenos Aires, Instituto de Física del Plasma (INFIP), Buenos Aires, Argentina

E-mail: minotti@df.uba.ar
} 
Revaluation of Mbelek and Lachièze-Rey scalar tensor theory of gravitation to explain the measured forces in

\section{Introduction}

The scalar-tensor (ST) gravitational theory of Mbelek and Lachièze-Rey (MLR) [3], has the particularity of allowing electromagnetic (EM) fields to modify the space-time metric far more strongly than predicted by General Relativity and standard ST theories. The theory was applied in cosmological [4] and galactic [5] contexts, and in [3] it was used to explain the discordant measurements of Newton gravitational constant as due to the effect of the earth's magnetic field. In [1] it was further shown that a ST theory of the MLR type could explain the unusual forces on asymmetric resonant cavities reported at that time [6]. However, in the derivation of the equations from the action principle some inconsistencies were observed, and also the forces obtained, after reconsideration of the experiments originally reported and new results [2], were too high. In the present work the equations are re-derived using the full variation of the action, and the constant of the theory re-evaluated with the consideration that no anomalous gravitational effects are produced by the earth's magnetic field. It is shown that the originally employed equations were correct, and that employing the new evaluated constant a correct magnitude for the forces reported recently is obtained.

\section{The MLR ST theory}

The details of the considered MLR ST theory were already presented in [1] and [7], so that only a brief account will be given here. The MRL action is given by (SI units are used)

$$
\begin{aligned}
S= & -\frac{c^{3}}{16 \pi G_{0}} \int \sqrt{-g} \phi R d \Omega+\frac{c^{3}}{16 \pi G_{0}} \int \sqrt{-g} \frac{\omega(\phi)}{\phi} \nabla^{\nu} \phi \nabla_{\nu} \phi d \Omega \\
& +\frac{c^{3}}{16 \pi G_{0}} \int \sqrt{-g} \phi\left[\frac{1}{2} \nabla^{\nu} \psi \nabla_{\nu} \psi-U(\psi)-J \psi\right] d \Omega \\
& -\frac{\varepsilon_{0} c}{4} \int \sqrt{-g} \lambda(\phi) F_{\mu \nu} F^{\mu \nu} d \Omega-\frac{1}{c} \int \sqrt{-g} j^{\nu} A_{\nu} d \Omega \\
& +\frac{1}{c} \int \mathcal{L}_{\text {mat }} d \Omega .
\end{aligned}
$$

In (11) the internal, non-dimensional scalar field is $\phi$, while $\psi$ is an external scalar field introduced by MRL to stabilize the reduced action of Kaluza-Klein multidimensional theories. These fields have vacuum expectation values (VEV) $\phi_{0}$ and $\psi_{0}$, respectively. $G_{0}$ is Newton gravitational constant, with $G_{0} / \phi$ being its value in vacuum (this means that $\left.\phi_{0}=1\right), c$ is the velocity of light in vacuum, and $\varepsilon_{0}$ is the vacuum permittivity. $\mathcal{L}_{\text {mat }}$ is the lagrangian density of matter. The other symbols are also conventional, $R$ is the Ricci scalar, and $g$ the determinant of the metric tensor $g_{\mu \nu}$. The Brans Dicke parameter $\omega(\phi)$ is considered a function of $\phi$. The function $\lambda(\phi)$ in the term of the action of the EM field is found in reduced, effective theories [4]. The EM tensor is $F_{\mu \nu}=\nabla_{\mu} A_{\nu}-\nabla_{\nu} A_{\mu}$, given in terms of the EM quadri-vector $A_{\nu}$, with sources given by the quadri-current $j^{\nu}$. $U$ and $J$ are, respectively, the potential and source of the 
Revaluation of Mbelek and Lachièze-Rey scalar tensor theory of gravitation to explain the measured forces in

field $\psi$. The source $J$ contains contributions from the matter, EM field and the scalar $\phi$. The model for $J$ proposed in [3] is

$$
J=\beta_{m a t}(\psi, \phi) \frac{8 \pi G_{0}}{c^{4}} T^{m a t}+\beta_{E M}(\psi, \phi) \frac{4 \pi G_{0} \varepsilon_{0}}{c^{2}} F_{\mu \nu} F^{\mu \nu},
$$

where $T^{\text {mat }}$ is the trace of the energy-momentum tensor of matter,

$$
T_{\mu \nu}^{m a t}=-\frac{2}{\sqrt{-g}} \frac{\delta \mathcal{L}_{m a t}}{\delta g^{\mu \nu}} .
$$

The $\beta$ coefficients are unknown functions of the scalars, but in the weak-field (WF) approximation they only contribute through the values of their first-order derivatives at the VEV $\phi_{0}$ and $\psi_{0}$, and thus appear as adjustable constants. Variation of (11) with respect to $g^{\mu \nu}$ results in ( $T_{\mu \nu}^{E M}$ is the usual electromagnetic energy tensor)

$$
\begin{aligned}
\phi\left(R_{\mu \nu}-\frac{1}{2} R g_{\mu \nu}\right)= & \frac{8 \pi G_{0}}{c^{4}}\left[\lambda(\phi) T_{\mu \nu}^{E M}+T_{\mu \nu}^{m a t}\right]+T_{\mu \nu}^{\phi} \\
& +\frac{\phi}{2}\left(\nabla_{\mu} \psi \nabla_{\nu} \psi-\frac{1}{2} \nabla^{\gamma} \psi \nabla_{\gamma} \psi g_{\mu \nu}\right) \\
& +\frac{\phi}{2}(U+J \psi) g_{\mu \nu}-\phi \psi \frac{\delta J}{\delta g^{\mu \nu}},
\end{aligned}
$$

where $T_{\mu \nu}^{\phi}$ is the energy tensor of the scalar $\phi$

$$
T_{\mu \nu}^{\phi}=\nabla_{\mu} \nabla_{\nu} \phi-\nabla^{\gamma} \nabla_{\gamma} \phi g_{\mu \nu}+\frac{\omega(\phi)}{\phi}\left(\nabla_{\mu} \phi \nabla_{\nu} \phi-\frac{1}{2} \nabla^{\gamma} \phi \nabla_{\gamma} \phi g_{\mu \nu}\right),
$$

and where, since $\delta T^{\text {mat }} / \delta g^{\mu \nu}=0$,

$$
\frac{\delta J}{\delta g^{\mu \nu}}=\frac{4 \pi G_{0} \varepsilon_{0}}{c^{2}} \beta_{E M}(\psi, \phi) F_{\mu \theta} F_{\nu \xi} g^{\theta \xi} .
$$

Variation with respect to $\phi$ gives

$$
\begin{aligned}
\phi R+2 \omega \nabla^{\nu} \nabla_{\nu} \phi= & \left(\frac{\omega}{\phi}-\frac{d \omega}{d \phi}\right) \nabla^{\nu} \phi \nabla_{\nu} \phi-\frac{4 \pi G_{0} \varepsilon_{0}}{c^{2}} \phi \frac{d \lambda}{d \phi} F_{\mu \nu} F^{\mu \nu} \\
& -\frac{\partial J}{\partial \phi} \psi \phi+\phi\left[\frac{1}{2} \nabla^{\nu} \psi \nabla_{\nu} \psi-U(\psi)-J \psi \phi\right],
\end{aligned}
$$

which can be rewritten, using the contraction of (3) with $g^{\mu \nu}$ to replace $R$, as

$$
\begin{aligned}
(2 \omega+3) \nabla^{\nu} \nabla_{\nu} \phi= & -\frac{d \omega}{d \phi} \nabla^{\nu} \phi \nabla_{\nu} \phi-\frac{4 \pi G_{0} \varepsilon_{0}}{c^{2}} \phi \frac{d \lambda}{d \phi} F_{\mu \nu} F^{\mu \nu} \\
& +\frac{8 \pi G_{0}}{c^{4}} T^{m a t}\left(1+2 \psi \phi \beta_{\text {mat }}\right)+\phi U(\psi) \\
& -\left(J+\frac{\partial J}{\partial \phi} \phi\right) \psi \phi,
\end{aligned}
$$

where it was used that $T^{E M}=T_{\mu \nu}^{E M} g^{\mu \nu}=0$. The non-homogeneous Maxwell equations are obtained by varying (1) with respect to $A_{\nu}$,

$$
\nabla_{\mu}\left\{\left[\lambda(\phi)+\phi \psi \beta_{E M}(\psi, \phi)\right] F^{\mu \nu}\right\}=\mu_{0} j^{\nu} .
$$


Revaluation of Mbelek and Lachièze-Rey scalar tensor theory of gravitation to explain the measured forces in with $\mu_{0}$ the vacuum permeability. Finally, the variation with respect to $\psi$ results in

$$
\nabla^{\nu} \nabla_{\nu} \psi+\frac{1}{\phi} \nabla^{\nu} \psi \nabla_{\nu} \phi=-\frac{\partial U}{\partial \psi}-\left(J+\frac{\partial J}{\partial \psi} \psi\right) .
$$

\section{Analysis}

A crucial point is indicated by Maxwell equations (5) because it turns out that the expected magnitude of $\phi \psi \partial \beta_{E M}(\psi, \phi) / \partial \phi$ at the VEV of the scalar fields is very large, which would clearly result in the wrong Maxwell equations. First, we note that, in order to recover Einstein-Maxwell equations when the scalar fields are not excited, the potential $U(\psi)$ and its derivative are zero when evaluated at the VEV $\psi_{0}, \lambda\left(\phi_{0}\right)=1$, and

$$
\beta_{\text {mat }}\left(\psi_{0}, \phi_{0}\right)=\beta_{E M}\left(\psi_{0}, \phi_{0}\right)=0 .
$$

One can then readily conclude that when the scalar fields are close to their VEV the correct Maxwell equations are obtained only if

$$
\nabla_{\mu} \psi=-\frac{\partial\left(\psi \phi \beta_{E M}\right) / \partial \phi}{\partial\left(\psi \phi \beta_{E M}\right) / \partial \psi} \nabla_{\mu} \phi \equiv h(\psi, \phi) \nabla_{\mu} \phi .
$$

In this relation we have left out the function $\lambda(\phi)$, which is responsible for possible effects of the scalar field on the electromagnetic fields [7]. Employing relation (8) in Eq. (6) we obtain

$$
\begin{aligned}
\nabla^{\nu} \nabla_{\nu} \phi= & -\left(\frac{1}{h} \frac{\partial h}{\partial \phi}+\frac{\partial h}{\partial \psi}+\frac{1}{\phi}\right) \nabla^{\nu} \phi \nabla_{\nu} \phi \\
& -\frac{1}{h}\left(\frac{\partial U}{\partial \psi}+J+\psi \frac{\partial J}{\partial \psi}\right),
\end{aligned}
$$

which is to be compared with Eq. (4). The first thing to note is that this implies that

$$
\frac{1}{h} \frac{\partial h}{\partial \phi}+\frac{\partial h}{\partial \psi}+\frac{1}{\phi}=\frac{d \omega / d \phi}{2 \omega+3} .
$$

Since the right-hand side is a function of only $\phi$, for reasons to be made clear immediately, it is expected that at the scalars VEV

$$
\frac{1}{h} \frac{\partial h}{\partial \phi}+\frac{\partial h}{\partial \psi}=0
$$

which, employing the definition of $h$ in (8) , is written as

$$
2 h \frac{\partial^{2}\left(\psi \phi \beta_{E M}\right)}{\partial \phi \partial \psi}+\frac{\partial^{2}\left(\psi \phi \beta_{E M}\right)}{\partial \phi^{2}}+h^{2} \frac{\partial^{2}\left(\psi \phi \beta_{E M}\right)}{\partial \psi^{2}}=0 .
$$

With the condition (7), relation (11) is satisfied at the scalars VEV if all second derivatives of $\beta_{E M}$ are zero at those scalar values. This is a self-consistent condition that also means that the equations around these values can be determined at second 
Revaluation of Mbelek and Lachièze-Rey scalar tensor theory of gravitation to explain the measured forces in

order in the perturbed scalars with the only parameter of the first derivatives of $\beta_{E M}$ at $\left(\psi_{0}, \phi_{0}\right)$. In this way we obtain from (10)

$$
\frac{\omega_{0}^{\prime}}{2 \omega_{0}+3}=1
$$

where $\omega_{0} \equiv \omega\left(\phi_{0}\right), \omega_{0}^{\prime} \equiv(d \omega / d \phi)_{\phi_{0}}$. Also, from the comparison between (44) and (9),

$$
\begin{aligned}
& \left.\frac{\psi_{0}}{h_{0}} \frac{\partial \beta_{m a t}}{\partial \psi}\right|_{\left(\psi_{0}, \phi_{0}\right)}=\frac{1}{2 \omega_{0}+3}\left(\left.\psi_{0} \frac{\partial \beta_{m a t}}{\partial \phi}\right|_{\left(\psi_{0}, \phi_{0}\right)}-1\right), \\
& \left.\frac{\psi_{0}}{h_{0}} \frac{\partial \beta_{E M}}{\partial \psi}\right|_{\left(\psi_{0}, \phi_{0}\right)}=\frac{1}{2 \omega_{0}+3}\left(\left.\psi_{0} \frac{\partial \beta_{E M}}{\partial \phi}\right|_{\left(\psi_{0}, \phi_{0}\right)}+\lambda_{0}^{\prime}\right),
\end{aligned}
$$

where $\lambda_{0}^{\prime} \equiv(d \lambda / d \phi)_{\phi_{0}}$, and

$$
h_{0}=-\left.\frac{\partial \beta_{E M} / \partial \phi}{\partial \beta_{E M} / \partial \psi}\right|_{\left(\psi_{0}, \phi_{0}\right)} .
$$

With the assumption that the dominant term in the bracket on the right-hand side of the second of (13) is the first one (the consistency is checked below), one obtains

$$
\left.\frac{\partial \beta_{E M}}{\partial \psi}\right|_{\left(\psi_{0}, \phi_{0}\right)}= \pm\left.\frac{1}{\left|2 \omega_{0}+3\right|^{1 / 2}} \frac{\partial \beta_{E M}}{\partial \phi}\right|_{\left(\psi_{0}, \phi_{0}\right)},
$$

with the additional condition that $2 \omega_{0}+3<0$. As is well known, this condition would lead to an unstable action for a pure Brans-Dicke theory. However, note that from (14) one has $h_{0}=\mp\left|2 \omega_{0}+3\right|^{1 / 2}$, which, together with relation (8), means that the MRL action (11) has, "on-shell", and at least around the scalars VEV, an effective positive Brans-Dicke parameter, which solves the instability problem, and what motivated the introduction of the external escalar field in MRL theory.

\section{Weak-field approximation}

With the considerations given above, Eq. (3) can be approximated in the WF limit keeping only the lowest significant order in the perturbations $h_{\mu \nu}$ of the metric $g_{\mu \nu}$ about the Minkowski metric $\eta_{\mu \nu}$, with signature $(1,-1,-1,-1)$, and of the scalar fields about their VEV $\phi_{0}$ and $\psi_{0}$, as

$$
-\eta^{\gamma \delta} \partial_{\gamma \delta} \bar{h}_{\mu \nu}=2\left(\partial_{\mu \nu} \phi-\eta^{\gamma \delta} \partial_{\gamma \delta} \phi \eta_{\mu \nu}\right),
$$

with the Lorentz gauge

$$
\partial_{\gamma} \bar{h}_{\nu}^{\gamma}=0
$$

where

$$
\bar{h}_{\mu \nu} \equiv h_{\mu \nu}-\frac{1}{2} h \eta_{\mu \nu},
$$


Revaluation of Mbelek and Lachièze-Rey scalar tensor theory of gravitation to explain the measured forces in with $\bar{h}=\eta^{\mu \nu} \bar{h}_{\mu \nu}$. The WF equations for the scalars considering only the electromagnetic sources are then

$$
\eta^{\gamma \delta} \partial_{\gamma \delta} \phi=-\Gamma F_{\mu \nu} F^{\mu \nu},
$$

and

$$
\eta^{\gamma \delta} \partial_{\gamma \delta} \psi= \pm\left|2 \omega_{0}+3\right|^{1 / 2} \Gamma F_{\mu \nu} F^{\mu \nu},
$$

with

$$
\Gamma=\left.\frac{4 \pi G_{0} \varepsilon_{0}}{c^{2}} \frac{\psi_{0}}{2 \omega_{0}+3} \frac{\partial \beta_{E M}}{\partial \phi}\right|_{\left(\psi_{0}, \phi_{0}\right)} .
$$

As can be seen in [1, for slow moving neutral masses, the WF limit of the geodesic equation corresponds to motion in flat Minkowski space-time under the action of a specific force (per unit mass) given by (Latin indices correspond to the spatial coordinates)

$$
f_{i}=-\frac{c^{2}}{4} \frac{\partial}{\partial x_{i}}\left(\bar{h}_{00}+\bar{h}_{k k}\right)+c \frac{\partial \bar{h}_{0 i}}{\partial t} .
$$

Using Eqs. (15) and (19) the gravitational field is thus represented in the WF approximation by a gravitational potential $\chi$ in flat Minkowski space-time, whose determining equation with only scalar field sources is $\left(\square \equiv \eta^{\gamma \delta} \partial_{\gamma \delta}\right)$

$$
\begin{aligned}
\square \chi & =\frac{\partial^{2} \phi}{\partial t^{2}}-\frac{c^{2}}{2} \square \phi \\
& =\frac{\partial^{2} \phi}{\partial t^{2}}+\frac{c^{2} \Gamma}{2} F_{\mu \nu} F^{\mu \nu},
\end{aligned}
$$

where (17) was used to write the second line.

\section{Constant determination}

As was argued in [1, in order for the MLR theory to be consistent with the lack of strong gravitational effects due to the magnetic field of the Earth, the non-linear terms in Eq. (4) should be kept, even in the WF approximation. This is so because the laplacian terms in these equations can be zero for this particular type of source, and thus the equalities are satisfied by the higher order terms. Specifically, for a static magnetic field outside its sources one can write $\mathbf{B}=\nabla \Psi$, with $\nabla^{2} \Psi=0$, so that, from Eq. (44), one has (here we use the consistency condition that the second derivatives of $\beta_{E M}(\psi, \phi)$ are zero at the VEV values)

$$
\nabla^{2} \phi+\frac{\omega_{0}^{\prime}}{2 \omega_{0}+3} \nabla \phi \cdot \nabla \phi=2 \Gamma B^{2},
$$

Eq. (21) has the solution, using also relation (12),

$$
\phi=1+\sqrt{2 \Gamma} \Psi .
$$

Noting that the measured gravitational constant is given by $G=G_{0} / \phi$, one can repeat the analysis made in [3] using expression (22) to obtain the values of $G_{0}$ and $\Gamma$ that 
Revaluation of Mbelek and Lachièze-Rey scalar tensor theory of gravitation to explain the measured forces in

gives the best fit between the locally measured value of $G$ and $G_{0} / \phi$, with $\phi$ given by (22) with the local value of the earth's magnetic field given in terms of $\Psi$ expressed, using Gauss coeffcients, by [3]

$$
\Psi=\frac{a^{3}}{r^{2}}\left(g_{1}^{0} \cos \theta+g_{1}^{1} \sin \theta \cos \varphi+h_{1}^{1} \sin \theta \sin \varphi\right),
$$

where $\varphi$ is the longitude, $\theta$ the co-latitude, $r$ the distance to the earth center, and $a$ the earth radius. Employing the same database and parameters in (23) as in [3] we obtain (SI units)

$$
\begin{aligned}
& G_{0}=6.6752 \times 10^{-11} \mathrm{Nm}^{2} \mathrm{~kg}^{-2}, \\
& \Gamma=3.35 \times 10^{-12} \mathrm{~A}^{2} \mathrm{~N}^{-2} .
\end{aligned}
$$

Note that, from (18), with this value of $\Gamma, \phi \psi \partial \beta_{E M} / \partial \phi$ at the scalars VEV has a very large value, as assumed. It is worth noting that this evaluation of $\Gamma$ is not completetly satisfactory because there are not enough data and with sufficient spread of the magnetic field values at the sites where $G$ was measured to have a reliable statistics. It is made here only to check that the theory can be consistent with different experimental results.

\section{Force evaluation}

In this section we present the basis for the evaluation of the force on axially symmetric cavities, including an expression more convenient than that used in [1] because the involved derivatives can be performed explicitly so that the final expression reduces to a (five-dimensional) integral. From what was seen in the previous section, in the weak field approximation, the average thrust is determined as a gravitational force derived from the gravitational potential $\chi$, whose determining equation is obtained from Eq. (20) by taking the time average

$$
\nabla^{2} \chi=-\varkappa \Sigma
$$

where the constant $\varkappa$ has the value (SI units)

$$
\varkappa \equiv c^{2} \Gamma=3.0 \times 10^{5} \mathrm{~A}^{2} \mathrm{~s}^{2} \mathrm{~kg}^{-2},
$$

and the source $\Sigma$ is the time average of the field invariant $B^{2}-E^{2} / c^{2}$

$$
\Sigma=\left\langle B^{2}-E^{2} / c^{2}\right\rangle .
$$

The obtained value of $\varkappa$ is a factor $10^{-4}$ of that used in [1. In this way, if (24) is employed to determine the force on the asymmetric resonant cavity employed in [2], one obtains forces of the order of $\mu \mathrm{N} / \mathrm{W}$, as those reported. If $\rho$ is the mass density of the material of the cavity the force on a cavity with thin walls is then given by

$$
\mathbf{F}=-\rho \int \lambda \nabla \chi d S
$$


Revaluation of Mbelek and Lachièze-Rey scalar tensor theory of gravitation to explain the measured forces in

where $\lambda$ is the local thickness of the cavity wall, and the surface integral is extended to the whole surface of the cavity. If the cavity and field distribution have axial symmetry, using spherical coordinates with the angle $\theta$ measured from the cavity axis, the solution of (24) can be written as

$$
\begin{aligned}
\chi(r, \theta)= & \frac{\varkappa}{\pi} \int \frac{\Sigma\left(r^{\prime}, \theta^{\prime}\right)}{\sqrt{r^{2}+r^{\prime 2}-2 r r^{\prime} \cos \left(\theta^{\prime}-\theta\right)}} \\
& \times K\left(-\frac{4 r r^{\prime} \sin \theta \sin \theta^{\prime}}{r^{2}+r^{\prime 2}-2 r r^{\prime} \cos \left(\theta^{\prime}-\theta\right)}\right) r^{\prime 2} \sin \theta^{\prime} d r^{\prime} d \theta^{\prime}
\end{aligned}
$$

where $K$ is the complete elliptic integral of the first kind, and the volume integral is extended to the interior of the cavity. For the same axial symmetry, the force (26) has component only along the direction of the cavity axis, which we call $z$, and is given by

$$
F_{z}=-\varrho \int \lambda(r, \theta)\left(\cos \theta \frac{\partial \chi}{\partial r}-\frac{\sin \theta}{r} \frac{\partial \chi}{\partial \theta}\right) d S .
$$

Using expression (27) in (28) the derivatives can be explicitly made and one finally has

$$
\begin{aligned}
F_{z}= & -\frac{\varkappa \rho}{\pi} \int \frac{\lambda(r, \theta) \Sigma\left(r^{\prime}, \theta^{\prime}\right)\left(r^{\prime} \cos \theta^{\prime}-r \cos \theta\right)}{\left[r^{2}+r^{\prime 2}-2 r r^{\prime} \cos \left(\theta^{\prime}+\theta\right)\right] \sqrt{r^{2}+r^{\prime 2}-2 r r^{\prime} \cos \left(\theta^{\prime}-\theta\right)}} \\
& \times E\left(-\frac{4 r r^{\prime} \sin \theta \sin \theta^{\prime}}{r^{2}+r^{\prime 2}-2 r r^{\prime} \cos \left(\theta^{\prime}-\theta\right)}\right) r^{\prime 2} \sin \theta^{\prime} d r^{\prime} d \theta^{\prime} d S
\end{aligned}
$$

where $E$ is the complete elliptic integral of the second kind. The integration over the primed variables is extended to the volume of the cavity, while the non-primed variables are integrated over the surface of the cavity. If a single electromagnetic mode, axially symmetric is considered, with angular frequency $\omega$, the field invariant $B^{2}-E^{2} / c^{2}$ can be written in general as

$$
B^{2}-E^{2} / c^{2}=F_{B}(r, \theta) \cos ^{2}(\omega t)-F_{E}(r, \theta) \sin ^{2}(\omega t),
$$

and so its time average is given simply by

$$
\Sigma=\left\langle B^{2}-E^{2} / c^{2}\right\rangle=\frac{1}{2}\left(F_{B}-F_{E}\right) .
$$

As done in [1] the cavity is approximated by a cone whose axis is taken as the $z$ direction. The lateral wall corresponds to the spherical angle $\theta=\theta_{0}$ (half angle of the cone), and the spherical caps to the radii $r=r_{1,2}$, with $r_{2}>r_{1}$. The resonant modes are standing electromagnetic waves satisfying the vector wave equation ( $\mathbf{F}$ stands for either the electric field $\mathbf{E}$, or the magnetic induction $\mathbf{B}$ )

$$
\frac{1}{c^{2}} \frac{\partial^{2} \mathbf{F}}{\partial t^{2}}-\nabla^{2} \mathbf{F}=0 .
$$

The modes with axial symmetry and $\mathbf{B}$ transverse to the $z$ direction $\mathbf{e}_{z}$ (called the TM modes) that satisfy this equation are (spherical coordinates are employed, with unit 
Revaluation of Mbelek and Lachièze-Rey scalar tensor theory of gravitation to explain the measured forces in vectors $\mathbf{e}_{r}, \mathbf{e}_{\theta}$ and $\left.\mathbf{e}_{\varphi}\right)$

$$
\begin{aligned}
\mathbf{B}= & -C k R(r) Q^{\prime}(\theta) \cos (\omega t) \mathbf{e}_{\varphi}, \\
\mathbf{E} / c= & C\left\{\frac{R(r)}{r} n(n+1) Q(\theta) \mathbf{e}_{r}\right. \\
& \left.+\left[\frac{R(r)}{r}+R^{\prime}(r)\right] Q^{\prime}(\theta) \mathbf{e}_{\theta}\right\} \sin (\omega t)
\end{aligned}
$$

where $C$ is a global constant. The complementary set of modes with $\mathbf{E}$ transverse to the $z$ direction (TE modes) have the expressions (30) and (31), but with $\mathbf{E} / c$ and $\mathbf{B}$ interchanged. The functions $R$ and $Q$ are defined as

$$
\begin{aligned}
& Q(\theta)=P_{n}(\cos \theta), \\
& R(r)=R_{+}(r) \cos \alpha+R_{-}(r) \sin \alpha, \\
& R_{ \pm}(r)=\frac{J_{ \pm(n+1 / 2)}(k r)}{\sqrt{r}},
\end{aligned}
$$

where $P_{n}$ is the Legendre polynomial of order $n, J_{m}$ the Bessel function of the first kind of order $m$. The constants $\alpha$ and $k$ are determined along with the order $n$ by imposing the boundary conditions of zero normal component of the magnetic field and of zero tangential components of the electric field at the metallic walls. Finally, the quality factor of the cavity, $Q_{c a v}$, for each mode is conventionally defined as

$$
Q_{c a v} \equiv \frac{\omega\langle U\rangle}{\langle W\rangle},
$$

where $\omega=k c$ is the angular frequency of the mode, $\langle U\rangle$ is the temporal average of its electromagnetic energy, and $\langle W\rangle$ is the average dissipated power in the wall cavities. If the cavity is fed with an average electromagnetic power $P$, in the permanent regime one has $\langle W\rangle=P$, and so,

$$
\langle U\rangle=\frac{\int\left\langle B^{2}\right\rangle d V}{\mu_{0}}=\frac{Q_{c a v} P}{\omega},
$$

which allows to determine the global constant $C$, given the fed average power and the characteristics of the cavity for the considered mode.

The electromagnetic mode reported in 2] is the TM212, which has no axial symmetry, and thus the corresponding force cannot be simulated with (29) (the reported value is $1.2 \mu \mathrm{N} / \mathrm{W}$, directed toward the small end of the cone). For this reason we will instead present the results of the model for a few axially symmetric TM and TE modes.

The cavity employed in [2] is simulated here with corresponding caps of radius $31.22 \mathrm{~cm}$ and $54.69 \mathrm{~cm}$, and side walls inclined 14.8 degrees from the axial direction. The copper side walls have $0.6 \mathrm{~mm}$ thickness, while the caps are made of a thin copper layer $(35.56 \mu \mathrm{m})$ over a $1.6 \mathrm{~mm}$ thick PCB board.

The TM010 mode evaluated for a cavity of these dimensions has a frequency of $973.2 \mathrm{MHz}$ with a quality factor of 34,000 . The corresponding force evaluated with (29) has a magnitude of $0.69 \mu \mathrm{N} / \mathrm{W}$ and directed toward the small end of the cone. For the TM012 mode the frequency is $1,6856 \mathrm{MHz}$ and the quality factor 33,700. The 
Revaluation of Mbelek and Lachièze-Rey scalar tensor theory of gravitation to explain the measured forces in

force has a magnitude of $0.14 \mu \mathrm{N} / \mathrm{W}$, directed toward the large end of the cone. For the TE010 mode the frequency is $1,7309 \mathrm{MHz}$ and the quality factor 70,800 . The force has a magnitude of $0.11 \mu \mathrm{N} / \mathrm{W}$, directed toward the large end of the cavity. For the TE012 mode the frequency is $2,1322 \mathrm{MHz}$ and the quality factor 79,000 . The force has a magnitude of $0.011 \mu \mathrm{N} / \mathrm{W}$, directed toward the large end of the cavity.

From all these cases, only the TM010 mode has the same direction and a magnitude comparable (about 57\%) to the force reported for the TM212 mode. The rest of the cases have a force directed toward the large end of the cone, and magnitudes about $10 \%$ of those reported, with the exception of the TE012 mode that has a very low value, only $1 \%$. Cases in which the force changes direction and/or has very low magnitudes were previously reported, but not so thoroughly studied as the published case.

\section{Conclusions}

We have rederived the equations of MRL theory employing a full variation of the action, and estimated the constant of the WF limit of the theory, consistent with the lack of anomalous effects by the earth's magnetic field. In this respect it is important to ascertain that expression (22) is the pertinent solution of the non-linear equation (21). From this equation one can determine, for instance, that if the magnetic field does not diverge the normal derivative of $\phi$ across any surface must be continuous. Since also the normal component of the magnetic field must be continuous across any surface, the solution (22) satisfies the correct boundary conditions if the sources of the magnetic field are confined to relatively thin sheets of electric current. Also, one can argue that, of all possible solutions compatible with given boundary conditions, those with minimum energy (defined in some appropriate sense) should be those found in a real system. In this way it is expected that the solution that minimizes the gravitational forces (with their feedback effects on the material sources, for instance forcing the electric current to be distributed on thin sheets) is the one verified in practice. In this respect MRL theory can be of fundamental importance to our understanding of the generation of geophysical and astrophysical magnetic fields. Of course, an evaluation of the constant in a specifically designed and well controlled experiment should be desirable, the only purpose of the present estimation is to show the compatibility of the theory with all available related data

With all this, it can now be ascertained with more confidence that the theory is consistent and compatible with the reported effects.

[1] Minotti F. O. 2013, Grav. \& Cosmol. 19201.

[2] White H., March P., Lawrence J., Vera J., Sylvester A., Brady D., and Bailey P. 2016, J. Prop. Power DOI: 10.2514/1.B36120.

[3] Mbelek J. P. and Lachièze-Rey M. 2002, Grav. \& Cosmol. 8331.

[4] Mbelek J. P. and Lachièze-Rey M. 2003, Astron. Astrophys. 397803.

[5] Mbelek J. P. 2004, Astron. Astrophys. 424761.

[6] Yang .J, Wang Y.-Q., Ma Y.-J., Li P.-F., Yang L., Wang Y., and He G.-Q. 2013, Chin. Phys. B 22 050301. 
Revaluation of Mbelek and Lachièze-Rey scalar tensor theory of gravitation to explain the measured forces in

[7] Raptis T. E. and Minotti F. O. 2013, Class. Quantum Grav. 30235004. 